\title{
CRISPR/CAS 9 Mediated Treatment for UTIS
}

\author{
Sarika Chaturvedi ${ }^{1} \mid$ Jinny Tomar $^{2}$ \\ 1,2Amity Institute of Biotechnology, Amity University, Gurugram, (Haryana)
}

To Cite this Article

Sarika Chaturvedi and Jinny Tomar, "CRISPR/CAS 9 Mediated Treatment for UTIs", International Journal for Modern Trends in Science and Technology, Vol. 06, Issue 05, May 2020, pp.: 82-94; https://doi.org/10.46501/IJMTST060515

\section{Article Info}

Received on 20-March-2020, Revised on 24-April-2020, Accepted on 02-May-2020, Published on 07-May-2020.

Copyright (C) 2014-2020 International Journal for Modern Trends in Science and Technology

DOI: https://doi.org/10.46501/IJMTST060515

\section{WHAT IS CRISPR TECHNOLOGY?}

"CRISPR" is short and used for "CRISPR-Cas9. CRISPR stands for clustered regularly interspaced short palindromic repeats. CRISPRs are specialized stretches of DNA. The protein Cas9 (or "CRISPR-associated") is an enzyme that acts like a pair of molecular scissors, capable of cutting strands of DNA and can be used in conjunction with engineered CRISPR sequences to hunt down codes and slice into them like a molecular scalpel, allowing geneticists to cut out a target gene, either to remove it or replace it with a new sequence. Therefore it is a simple and powerful tool for editing genomes to easily alter DNA sequences and amend gene function. In 1987, The CRISPR locus was first identified in Escherichia coli and discovered when a genetic structure containing 5 highly homologous repeats of 29 nucleotides separated by 32-nucleotide spacers (Ishino Y 1987).

\section{HOW CRISPR IS USED?}

CRISPR-based technology has been applied to correcting from removing genes responsible for diseases, to destroying drug resistant superbugs, to creating molecular recording devices, genetic defects, treating and preventing the spread of diseases and improving crops. This technology is used to target specific stretches of genetic code to edit the DNA at specific locations, drug development, agriculture, mutagenic animals, treating humans with genetic disorders etc. This system is found in bacteria which prevent it from invading viruses.
These sequences play a key role in the antiviral (i.e. anti-phage) defense system of prokaryotes (Barrangou R 2015). When viruses enter a bacterium it produces two short RNA's in which one of the RNA contains matching sequence of the virus and it is also known as "guide RNA". These guide sequence attaches to a specific target sequence of DNA in a genome as well as also binds to Cas9 enzyme and other enzymes as Cpf1. Then cells own DNA repair mechanisms add and delete pieces of genetic material to make changes to the DNA by replacing an existing segment with a customized DNA sequence. However, CRISPR system can also work with Cpf1 enzyme forming CRISPR-Cpf1.

Cpf1 is different from Cas9 in many ways like

- It requires only a single RNA for making the complex.

- It is smaller than Cas9 that is easier to deliver into cells and tissues.

- It cuts the DNA in different manner unlike Cas9 it cuts the both the strands at the same place.

- It has different PAM sites however, it also requires a PAM site initially and the target site is chosen adjacent to the PAM site (Xiao et al 2019)

This technology was adapted from the natural defense mechanisms of bacteria and archaebacteria. It was adapted from a naturally occurring genome editing system in bacteria. The bacteria capture oddments of DNA from invading viruses and use them to create DNA segments known as CRISPR arrays. The CRISPR arrays allow 
the bacteria to "remember" the viruses (or closely related ones). If the viruses attack again, the bacteria produce RNA segments from the CRISPR arrays to target the viruses' DNA. The bacteria then use Cas 9 or a similar enzyme to cut the DNA apart, which disables the virus.

\section{CRISPR APPLICATION TO INFECTIOUS Diseases}

CRISPR-Cas Technology has mainly application in the field of infectious disease $\mathrm{s}$ as today infectious diseases a global threat to excess morbidity and death annually, with the persistent potential for destabilizing pandemics. This technology provides tools for host and microbe interactions, to aid in the development of rapid and accurate diagnostics and to advance the prevention and treatment of infectious diseases. CRISPR-Cas9-based gene editing has been used across diverse pathogens to inform gene and protein contributions to molecular pathogenesis, host-pathogen interactions to develop accurate and portable diagnostic tests, and to advance the prevention and treatment of infectious diseases (Wei, X 2019). CRISPR function as an adaptive immune system for prokaryotes protecting them against foreign invaders and contributing to advances in biomedical sciences (Strich JR 2019) Acquired Immuno Deficiency Syndrome (AIDS) caused by infection of HIV remains a major threat to global human health. CRISPR) /CRISPR-associated nuclease 9 (Cas9) system has been engineered as an effective gene-editing technology with the potential to treat AIDS. It can be used to target cellular co-factors or HIV-1 genome to decrease HIV-1 infection and clear the provirus, as well as to provoke transcriptional activation of latent virus in latent viral reservoirs for exclusion. This versatile gene editing technology has been successfully applied to HIV-1/AIDS prevention and reduction in human cells and animal models (Qiaoqiao Xiao 2019).

Likewise in case of Urinary Tract Infections (UTIs), it has been observed that the number of community acquired cases as well as hospital acquired cases (HAIs) have been continuously increasing due to the presence of multi drug resistant (MDR) and extensive drug resistant (XDR) species and strains (Lima et al 2019). Being a hot topic from the past few decades, UTI has been found to be one of the most important contributor of not only female health issues but infants as well as male population of different age groups.

UTI also causes morbidity in individuals with urinary tract abnormalities. It may also induce pre term births, renal damage, sepsis. UTIs can be symptomatic (frequent urination, burning sensation, pain during urination, lower abdominal pain) or non symptomatic, symptomatic UTI may include presence of higher levels of bacteria in urine (bacteriuria), inflammation of urethra (urethritis), infection induced inflammation of urinary bladder (cystitis) and kidney (pyelonephritis). The reoccurrence and lack of timely treatment may worsen the situation leading to infection of the kidneys (O'brien et al 2016).

The increasing morbidity has been found to be due to etiologic agents like E. coli, Streptococcus, Klebsiella, Pseudomonas spp. and Enterococcus strains (Zuberi et al 2017).

Thus, the study by Ma et al, 2019, have given these pathogens a name-ESKAPE (Enterococcus faecium, Staphylococcus aureus, Klebsiella pneumoniae, Acinetobacter baumannii, Pseudomonas aeruginosa, and Enterobacter species) in their study based on their adaptability to escape effects of antibiotics.

These members of ESKAPE have been able to depict range of resistance, starting from target modification, changes in efflux pump expression, alteration in cell permeability, inducing enzymatic inactivation and so on.

Thus different combinatorial drugs have been actively used to regulate the ESKAPE pathogens, as the first generation drug- vancomycin and the second generation drugs - dalbavancin, oritavancin and oxazolidinone antibiotics have not been able to completely regulate these pathogens (Ma et al 2019).

Although, a series of new drugs have been approved by FDA after the successful completion of clinical trials like- Finafloxacin (fluoroquinolone), Plazomicin (semi synthetic aminoglycoside), Eravacycline (synthetic fluorocycline), Omadacycline (aminomethycycline) (Scott, 2019) Another approach is CRISPR-Cas, where as per our knowledge the two main components are Cas proteins and CRISPR RNA (crRNA). It has been seen that crRNA mediates the binding of target sequences with a region complementary to the target DNA. The role of Cas protein is to cleave the DNA and creates a double-strand DNA break (Chen et al 2019).

The CRISPR-Cas system can be classified into six types, the two main classes: class 1 and class 2 are based on phylogenetic relationship and activity mechanisms (Koonin et al., 2017). Class 1 systems includes types I, III, and IV and also contains effector complexes containing multiple Cas 
proteins. Class 2 systems on the other hand includes types II, V, and VI, employ effector complexes containing a single Cas protein to cleave the target DNAs (Koonin et al 2017) (Chen et al 2019).

There is a Type III of CRISPR-Cas system seen in case of Staphylococcus, where the CRISPR-Cas 10 system of Staphylococcus were supported by the crRNA from an exogenous plasmid This type III system was found effective in treatment of virulent strains of staphylococcus (Bari et al 2017).

As we know that CRISPR-Cas class II can provide a specific sequence based treatment which is very unlikely the traditional methods of treatment. Since the first study based on enterococcus has been able to explain that the enterococcus lacks CRISPR-CAS systems, thus there is a need to introduce CRISPR-Cas through proper vectors. The cascade is shown effective in selectively targeting genes involved in antibiotic resistance, bio film formation and even in reducing the virulence (Lee et al 2018).

In one of the study based on E. faecalis, phage can be used to deliver selected genes-CRISPR, but since the entire cascade is not present in enetrococcus spp. the effectiveness of the process was increased due to the pheromone responsive based plasmid (Prps) specifically for Cas9 gene transfer. Although a major reading was that in $\mathrm{E}$. faecalis the cells self target CRISPR-Cas system, targeting lethality specifically when the expression of Cas 9 was tried to increase (Rodrigues et al 2019).

\section{Concluding Remarks}

It is the growing resistance to $M D R / X D R$ that ESKAPE is emerging as a problem which needs immediate attention, and the choice of therapies for their treatment need to include new ways of transportation and delivery of drugs. Drug delivery system like the involvement of nano particles based delivery system, in certain cases phage based mode of delivery can also be explored in length.

Amongst the many boons, one point of concern associated with CRISPR-Cas associated phage therapy is the unintended activation of host immune responses. The lysis of the bacterial host, may releases endotoxins, which may further trigger an immune response, and such kind of implications cannot be ignored.

Thus more and more experimentations need to be conducted to optimize the gene cascade, with the sole aim of increasing the efficacy and specificity of CRISPR/Cas9 in target organisms, as there is still scarcity of published data. This can be further verified based on the clinical trials, which may also be able to rule out whether there exists any adverse effect due to off target the gene For CRISPR/Cas9 Another set of challenges in this path is in the manufacturing process as well as the approvals from regulatory authorities, for this constant efforts need to be made from drug developers to not only communicate but also demonstrate the safety and efficacy of phage based CRISPR-Cas therapy as a step towards personalised medicine with customisation as per the patient requirement.

\section{REFERENCES}

[1] Bari, S. M. N., Walker, F. C., Cater, K., Aslan, B., and Hatoum-Aslan, A. (2017). Strategies for editing virulent staphylococcal phages using CRISPRCas10. ACS Synth. Biol. 6, 2316-2325. doi: 10.1021/acssynbio.7b00240

[2] Barrangou R (2015). The roles of CRISPR-Cas systems in adaptive immunity and beyond". Current Opinion in Immunology. 32: 36-41.

[3] Chen Y, Batra H, Dong J, Chen C, Rao VB and Tao P (2019) Genetic Engineering of Bacteriophages Against Infectious Diseases. Front. Microbiol. 10:954. doi: 10.3389 /fmicb.2019.00954

[4] Ishino Y, Shinagawa H, Makino K, Amemura M, Nakata A 1987. Nucleotide sequence of the iap gene, responsible for alkaline phosphatase isozyme conversion in Escherichia coli, and identification of the gene product. J Bacteriol 169:5429-5433.

[5] Koonin, E. V., Makarova, K. S., and Zhang, F. (2017). Diversity, classification and evolution of CRISPR-Cas systems. Curr. Opin. Microbiol. 37, 67-78. doi: 10.1016/j.mib.2017.05.008

[6] Lee Hui Ling, Shen Haosheng, Hwang In Young, Ling Hua, Yew Wen Shan, Lee Yung Seng and Chang Matthew Wook,(2018) Targeted approaches for Insitu Gut Microbiome Manipulation, Genes:9, 351. Doi:10.3390/genes9070351

[7] Lima Renata, Fiol Fernando Sa Del and. Balcao Victor M,(2019) Prospects for the use of new technologies to combat MDR, frontiers in microbiology 10:692;doi:10.3389/fphar.2019.00692

[8] Ma Yu-Xuan, Wang Chen-Yu, Li Yuan-Yuan, Li Jing, Wan Qian-Qian, Chen Ji-Hua, Tay Franklin R. and Niu Li-Na, (2020) Considerations and Caveats in combating ESKAPE pathogens against Nosocomial Infections, Advanced Science:7; doi;10.1002/advs.201901872

[9] O’Brien Valerie P, Hannan Thomas J., Nielsen Hailyn V. and Hultgren Scott J., (2016) Microbiol Spectr. 4(1): doi:10.1128/microbiolspec.UTI-0013-2012.

[10] Rodrigues M, McBride SW, Hullahalli K, Palmer KL, Duerkop BA. 2019. Conjugative delivery of CRISPR-Cas9 for the selective depletion of antibiotic-resistant enterococci. Antimicrob Agents Chemother 63:e01454-19

[11] Scott L.J. Eravacycline: A Review in Complicated Intra - Abdominal Infections. Drugs. 2019;79(3):315-24

[12] Strich JR1, Chertow DS2,3 CRISPR-Cas Biology and Its Application to Infectious Diseases. J Clin Microbiol. 2019 Mar 28;57(4). pii: e01307-18. doi: 10.1128/JCM.01307-18. Print 2019 Apr.

[13] Qiaoqiao Xiao, Deyin Guo and Shuliang Chen Application of CRISPR/Cas9-Based Gene Editing in HIV-1/AIDS Therapy Front. Cell. Infect. Microbiol., 22 March 2019

[14] Wei, X. \& Nielsen, R. Nature Med. https://doi.org/ 10.1038/s41591-019-0459-6 (2019). 
[15] Zuberi A, Ahmad N and Khan AU (2017) CRISPRi Induced Suppression of Fimbriae Gene (fimH) of a Uropathogenic Escherichia coli: An Approach to Inhibit Microbial Biofilms. Front. Immunol. 8:1552. doi: 10.3389/fimmu.2017.01552

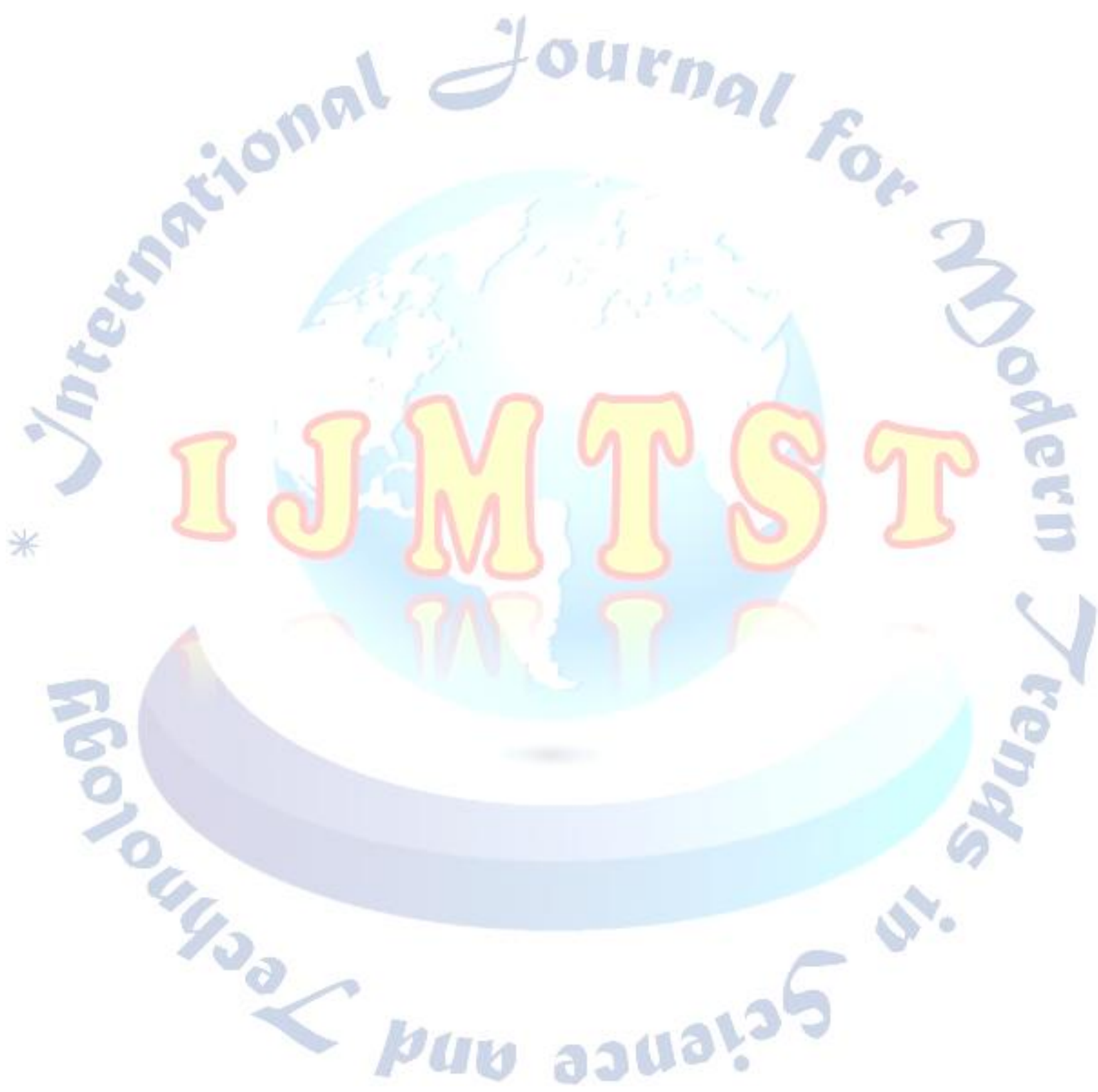

